\title{
Using Late Supplemental Oxygen to Prevent Retinopathy of Prematurity Progression In Premature Infants: a Retrospective Study
}

Robert Minturn ${ }^{1}$, Mary Beth Koch ${ }^{2}$, Emily Anderson ${ }^{2}$, Kok Lim Kua ${ }^{2}$, Kathryn Haider ${ }^{3}$

${ }^{1}$ Indiana University School of Medicine; ${ }^{2}$ Indiana University School of Medicine, Department of Neonatology- Perinatal Medicine; ${ }^{3}$ Indiana University School of Medicine, Department of Ophthalmology

\section{Background/Objective:}

Retinopathy of Prematurity (ROP) is a leading causes of childhood blindness. It affects 15,000 surviving US preterm infants annually, with 1,400 infants developing severe ROP and 500 infants developing legal blindness. The pathogenesis of ROP involves 2 phases: During phase 1 , the immature retinal vascularization is obliterated due to hyperoxia. During phase 2 (>4 weeks postnatally), abnormal neovascularization occurs due to hypoxia, sometimes requiring surgical intervention. We retrospectively evaluated the impact of late supplemental oxygen ( $>4$ weeks postnatally) on ROP progression in infants born $<28$ weeks.

\section{Methods:}

Preterm infants $<28$ weeks with >stage 2 ROP admitted to the Riley Hospital for Children Neonatal Intensive Care Unit (NICU) from 7/2017- 12/2019 were included. Nine patients treated with supplemental oxygen therapy were compared to a control cohort managed by a standard protocol after the diagnosis of stage 2 ROP. The primary outcome was the need for surgical intervention with either laser or bevacizumab treatment. Continuous data was analyzed using unpaired t-test, and categorical data was analyzed using fishers exact test.

\section{Results:}

There was no statistical difference in regard to clinical variables contributing to risk of severe ROP (sex, race, birthweight necrotizing enterocolitis, bronchopulmonary dysplasia or length of stay) between the two study cohorts. There was a statistically significant decrease in need for treatments (laser or bevacizumab) in patients receiving supplemental oxygen (control: 35/83 patients treated, late 02: 0/9 patients treated, $p=0.012$ ).

\section{Conclusion and Implications:}

Supplemental oxygen therapy seems to have a protective effect on the development of treatable ROP (type I). Limiting surgical intervention (laser or bevacizumab) would directly benefit the babies by decreasing the need for sedation and any inherent risks of surgery. This initial data suggests the need for future studies with a higher sample size to validate the efficacy of late supplemental $\mathrm{O} 2$ in ROP. 


\begin{tabular}{|c|c|c|c|c|c|}
\hline & \multicolumn{2}{|c|}{ Study Group } & \multicolumn{2}{|l|}{ Control } & \multirow[b]{2}{*}{$p$-value } \\
\hline & Number (Mean) & $\%(S D)$ & Number (Mean) & $\%(S D)$ & \\
\hline Population (N) & 9 & - & 83 & - & \\
\hline \multicolumn{6}{|l|}{ Sex } \\
\hline Male & 2 & $22.2 \%$ & 35 & $42.2 \%$ & N.S. \\
\hline Female & 7 & $77.8 \%$ & 48 & $57.8 \%$ & N.S. \\
\hline \multicolumn{6}{|l|}{ Race } \\
\hline Caucassian & 5 & $55.6 \%$ & 44 & $53.0 \%$ & N.S. \\
\hline Non-Caucassian & 4 & $44.4 \%$ & 39 & $47.0 \%$ & N.S. \\
\hline $\begin{array}{l}\text { Birthweight } \\
\text { (grams) }\end{array}$ & 698 & 145.26 & 721 & 197.09 & N.S. \\
\hline $\begin{array}{l}\text { Gestational Age } \\
\text { (Weeks) }\end{array}$ & 24.94 & 1.19 & 25.32 & 1.72 & N.S. \\
\hline $\begin{array}{l}\text { Length of Stay } \\
\text { (Days) }\end{array}$ & 129.22 & 27.13 & 148.58 & 67.97 & N.S. \\
\hline \multicolumn{6}{|l|}{$\begin{array}{l}\text { Necrotizing } \\
\text { Enterocolitis }\end{array}$} \\
\hline Yes & 3 & $33.3 \%$ & 21 & $25.3 \%$ & N.S. \\
\hline No & 6 & $66.7 \%$ & 62 & $74.7 \%$ & N.S. \\
\hline \multicolumn{6}{|l|}{ Sepsis } \\
\hline Yes & 6 & $66.7 \%$ & 41 & $49.4 \%$ & N.S. \\
\hline No & 3 & $33.3 \%$ & 42 & $50.6 \%$ & N.S. \\
\hline \multicolumn{6}{|c|}{$\begin{array}{l}\text { Bronchopulmonary } \\
\text { Dysplasia }\end{array}$} \\
\hline Yes & 9 & $100.0 \%$ & 80 & $96.4 \%$ & N.S. \\
\hline $\begin{array}{l}\text { No } \\
\text { Need for } \\
\text { Additional } \\
\text { Treatment } \\
\text { (Inpatient) }\end{array}$ & 0 & $0.0 \%$ & 2 & $2.4 \%$ & N.S. \\
\hline Yes & 0 & $0.0 \%$ & 35 & $42.2 \%$ & N.S. \\
\hline $\begin{array}{l}\text { No } \\
\text { Need for } \\
\text { Additional } \\
\text { Treatment } \\
\text { (Outpatient) }\end{array}$ & 9 & $100.0 \%$ & 48 & $57.8 \%$ & N.S. \\
\hline Yes & 0 & $0.0 \%$ & 35 & $42.2 \%$ & 0.012 \\
\hline No & 9 & $100.0 \%$ & 48 & $57.8 \%$ & \\
\hline
\end{tabular}

